\title{
NOTES AND COMMENTS \\ WHAT CHANCE DID MENDEL'S EXPERIMENTS GIVE HIM OF NOTICING LINKAGE?
}

\author{
L. DOUGLAS and E. NOVITSKI \\ Department of Genetics, University of Nijmegen, Nijmegen, The Netherlands \\ and \\ Department of Biology, University of Oregon, Eugene, Oregon 97403
}

Received 28.x.76

\section{SUMMARY}

The a priori probability of noticeable linkage among all conceivable experiments of the size reported by Mendel cannot reasonably be taken as greater than 24-36 per cent; and therefore, the frequently heard opinion that his chances of encountering linkage were high, approaching 99.4 per cent, appears to be mistaken.

\section{Introduction}

AN often heard criticism of Mendel (1886, translation in Sinnott et al., 1958, p. 419) is that he might have noticed linkage without reporting it. It is argued that he studied seven genes in a species with only seven chromosomes and that the a priori probability of choosing one gene on each chromosome is

$$
(6 / 7)(5 / 7) \ldots(1 / 7)=6 ! / 7^{6} \simeq 0 \cdot 006
$$

that is, the chance of a second gene on an unoccupied chromosome is $6 / 7$, of a third one, $5 / 7$, etc..

However, as indicated by Blixt (1975), (1) is an oversimplification in not taking genetic length of chromosomes into account. Moreover, it is also erroneous in assuming that Mendel made detailed studies on all pairs of factors which are possible using seven genes, which is $\left(\begin{array}{l}7 \\ 2\end{array}\right)=21$. Only 6 of these 21 were reported in detail in 1886; and although Mendel referrcd to crosses using the other (15) pairs, he mentioned that these were based on relatively small numbers of experimental plants.

Interesting also are comparisons of known genes in the garden pea, Pisum arvense (Blixt, 1974), with phenotypic descriptions published by Mendel (see Nilsson, 1951; Blixt, 1975; Novitski and Blixt, 1976). Although these comparisons do not permit unambiguous assignment of the seven factors to known loci, Mendel's published studies on two- and three-factor crosses seem to have involved four genes among which linkage is not detectable (Nilsson, 1951; Blixt, 1975). That is, Mendel may have had as many as 34 genetic stocks at his disposal, and just by chance, the four he used for two- or three-factor crosses are not noticeably linked. But how large is this chance? Is it so tiny as to suggest premeditated screening of stocks for factors which are not linked? Or is the absence of demonstrable linkage a reasonable outcome among all conceivable choices of four genes?

Earlier attempts to examine the credibility of Mendel's work were made at a time when linkage maps of the garden pea were incomplete or only 
fragmentary (e.g. see Johannsen, 1926; Fisher, 1936; Wright, 1966); but the maps reported by Blixt (1974) are fairly complete, giving genetic lengths of the seven chromosomes as 234, 204, 211, 247, 191, 59 and 109 centi Morgans $(=\mathrm{cM})$. If the seven maps are approximately linear, the sum $S \equiv 234+\ldots=1255$ suggests the probability density

$$
f(x)=1 / 1255, \quad 0 \leqq x \leqq 1255
$$

obtained by mapping line segments, with lengths proportional to those of the seven chromosomes, end-to-end on to the $x$-axis--so that the probability of a locus $x \mathrm{cM}$ from the origin in constant $=1 / S$.

The present attempt makes use of the density (2) as a rule for choosing loci at random; sets of such loci are then employed for constructing the $a$ priori probability $P(n, K)$ of choosing $n>2$ genes in such a way that none of them is linked more closely with any of the others than a preselected $K / 2 \mathrm{cM}$. Then by deciding on a $K$, or range of $K$ 's, which might reasonably have led Mendel to notice linkage, specific $P(n, K)$ 's of interest may be calculated (table 1). For example, it seems unlikely that a $K / 2$ between genes exceeding $30-50 \mathrm{cM}$ would have caused him to notice linkage. (Genes closer to each other than $K / 2 \mathrm{cM}$, and on the same chromosome, are referred to below as " detectably" or " noticeably" linked.)

\section{'TABLE 1}

\begin{tabular}{|c|c|c|}
\hline \multicolumn{3}{|c|}{$\begin{array}{l}\text { Probability of not observing linkage in the garden } \\
\text { pea, for experiments with } \mathrm{n}=2,3, \ldots, 7 \\
\text { genes and } \mathrm{K}=60 \text { or } 100\end{array}$} \\
\hline$n$ & 60 & 100 \\
\hline 2 & 0.956 & 0.931 \\
\hline 3 & 0.872 & 0.804 \\
\hline 4 & 0.758 & 0.638 \\
\hline 5 & 0.625 & 0.463 \\
\hline 6 & 0.488 & $0 \cdot 304$ \\
\hline 7 & $0 \cdot 360$ & 0.179 \\
\hline
\end{tabular}

\section{Results AND Discussion}

After $m=n-1$ non-linked genes are chosen using rule (2), several chromosome segments will be unavailable for the $n$th gene if independent assortment is to be maintained. Defining the total mean length of these segments as $T(m, K)$ (see the Appendix), the probability of choosing the $n$th gene in $(T)$, giving detectable linkage, becomes $T(m, K) / S$ and the probability of not doing so is $1-T / S$. From this, the probability of not choosing any of the $n$ genes closer to each other than $K / 2 \mathrm{cM}$ is simply

$$
P(n, K)=\prod_{m=1}^{n-1}[1-T(m, K) / S], \quad n=2,3, \ldots
$$

The exact expression for $T(m, K)$ is intricate, but for $n<7$, the error in (3) resulting from the approximation

$$
m T(1, K) \approx T(m, K)
$$


is not greater than 3 per cent (see the Appendix). Approximations o $P(n, K)$ obtained by substituting (4) into (3) are shown in table 1 . As seen, the probability of not observing linkage for $n=4$ is between 0.638 and 0.758 if $60<K<100$.

Blixt's (1975) assignments of the seven characters Mendel described to the garden pea map included two genes which are linked (le at 4,199 and pods smooth or wrinkled $=v$ at 4,211). However, Mendel did not explicitly report experiments testing $l e$ and $v$ together (even though he did mention testing all possible pairs of factors using small numbers of experimental plants-see Sinnott et al., 1958, p. 429). Several reasons for omission of $l e-v$ results are conceivable, one of which relates simply to the effort involved in studying large numbers of crosses for all of the $\left(\begin{array}{l}7 \\ 2\end{array}\right)=21$ pairs one may construct using seven genes. A more interesting speculation is suggested by Mendel's statement that, ". . . . in some forms the length of the stem [le] was very various" (brackets added, for the quotation see p. 422 in Sinnott et al., 1958). He did not express such uncertainty about classifying the other six pairs of phenotypes, and it therefore seems reasonable that he may not have studied $l e$ (or $l e-v$ ) as intensively as the other six. The alternative that he might even have had data suggesting $l e-v$ linkage, raises a question: in view of his doubts about the constancy of le phenotypes, would he have recognised the linkage as a distinct biological phenomenon or would he only have become even more skeptical about classifying le/le? It seems reasonable to speculate that he would have been skeptical-especially since $l e-v$ is the only pair, of the $\left(\begin{array}{l}7 \\ 2\end{array}\right)=21$ he could have constructed, giving " abberant" assortment (i.e. linkage).

Finally a point about the distribution of seven genes over seven chromosomes in the garden pea may be noted. If Mendel had deliberately screened his (34?) stocks in an attempt to find seven non-linked genes, a choice departing from one of the most probable ways to distribute seven genes over seven chromosomes might have occurred. This, in any case, is not suggested by table 2 , in which standard multinomial probabilities with $p_{i}=L_{i} / S$ corresponding to length, $L_{i}$, of the $i$ th chromosome, are displayed: according to Nilsson (1951) Mendel chose 2 genes on each of two chromosomes and 1 on each of three others. The probability of this is $P(2,2,1,1,1,0,0)=27 \cdot 4$ per cent approximately, which is the most probable of the 15 possible distributions. The distribution according to Blixt (1975) is 3, 2, 1, 1, 0, 0, 0 for which the probability is 24.9 per cent (approx.) -also one of the most probable.

\section{APPENDIX}

$T(1, K)$ is defined as the mean length of the chromosome segment about the first gene chosen, referred to $s_{1}$ below, in which a second must occur to give noticeable linkage (noticeable linkage implying that the two are nearer to each other than a pre-assigned $K / 2 \mathrm{cM}) . \quad T(1, K)$ is therefore the expectation

$$
T(1, K)=\frac{1}{S} \sum_{i=1}^{7} L_{i}\left[\frac{2}{L_{i}} \int_{0}^{L_{i} / 2} t(1, K, r, i) d r\right]
$$


TABLE 2

The probability of different ways to distribute seven genes over the seven chromosomes of the garden pea. In each case the probability is obtained from the standard multinomial

$$
P\left(x_{1}, \ldots, x_{7}\right)=\frac{7 !}{\Pi x_{i} !} \Sigma\left(\Pi p_{i}{ }^{x i}\right)
$$

where summation is on the permutation space $Q=Q\left(x_{1}, \ldots, x_{7}\right)$ such that the $\mathrm{x}_{\mathbf{i}} s$ in the superscripts are permuted while the $\mathrm{p}_{\mathrm{i}}$ remain in fixed sequence

$\begin{array}{cc}\text { Distribution } & P \times 1000 \\ 7000000 & 0 \cdot 0280 \\ 6100000 & 0 \cdot 882 \\ 5200000 & 2 \cdot 31 \\ 5110000 & 9 \cdot 77 \\ 4300000 & 3 \cdot 63 \\ 4210000 & 42 \cdot 7 \\ 4111000 & 47 \cdot 2 \\ 3310000 & 27 \cdot 4 \\ 3220000 & 37 \cdot 9 \\ 3211000 & 249 \cdot 5 \\ 3111100 & 101 \cdot 0 \\ 2221000 & 113 \cdot 9 \\ 2211100 & 274 \cdot 5 \\ 2111110 & 86 \cdot 2 \\ 1111111 & 3 \cdot 14\end{array}$

where $t$ is the length of $s_{1}$ about the first gene, which after being chosen using rule (2), is observed at a distance of $r \mathrm{cM}$ from a reference end of $i$ th chromosome. $L_{i}$ is the length in $\mathrm{cM}$ of the $i$ th chromosome and $\Sigma L=S$ (since $t$ is symmetric about $L_{i} / 2$, integration to the middle of the $i$ th linkage map and doubling is equivalent to integration completely to the limit $L_{i}$ ).

The procedure for assigning $t$ 's to different $r$ 's and $i$ 's is simple. For example if $L_{i}>K, t$ is

$$
t(1, K, r, i) \begin{cases}=r+K / 2 & 0<r<K / 2 \\ =K & K / 2<r<L / 2\end{cases}
$$

which brings the integral in A.1 to

For $K / 2<L_{i}<K$

$$
2 \int_{0}^{L / 2} t d r=K L_{i}-K^{2} / 4
$$

$$
t(1, K, r, i) \begin{cases}=r+K / 2 & 0<r<L-K / 2 \\ =L & L-K / 2<r<L / 2\end{cases}
$$

which also gives (A.3) after integrating. Since $L_{i}<K / 2$ does not occur in the garden pea, given reasonable choices of $K,(\mathrm{~A} .3)$ is valid for all seven chromosomes and (A.1) bccornes

$$
\begin{aligned}
T(1, K) & =\Sigma\left(K L_{i}-K^{2} / 4\right) / S \\
& =K-7 K^{2} / 4 S
\end{aligned}
$$

which is the desired result. 
$T(2, K)$ could be obtained using a procedure similar to the above, but is very nearly $2 T(1, K)$ (of course the segments $s_{1}$ and $s_{2}$ about the first and second genes may overlap, if on the same chromosome, without giving detectable linkage. This would cause $2 \mathcal{T}(1, K)$ to be slightly larger than $T(2, K))$. In general, it is easy to show that $T(n, K)$ for $n \leqq 7$ is, within a small error, approximately $n T(1, K)$.

Acknowledgments.- The authors are grateful to Prof. W. Seyffert, Prof. S. Geerts and Dr P. v.d. Kroon for careful criticism of the manuscript, to $\mathrm{Mr}$ J. Gerritsen for valuable comments and to the computer service at the University of Nijmegen for expeditious processing of input. The authors are also especially appreciative of the library materials provided by Dr J. Sybenga.

\section{RefERENGES}

BLIxt, s. 1974. The pea. In Handbook of Genetics, ed. R. C. King. Plenum Press, N.Y. BLIXT, s. 1975. Why didn't Gregor Mendel find linkage? Nature, 256, 206.

FISHER, R. A. 1936. Has Mendel's work been rediscovered? Ann. Sci., 1, 115-137.

JOHANnsen, w. 1926. Elemente der exakten Erblichkeitslehre. [See A History of Genetics (p. 15), ed. A. H. Sturtevant. Harper \& Row Pub., N.Y.]

MENDEL, G. 1886. Experiments in Plant Hybridization. (Reprinted in 1965 with an introduction by R. A. Fisher.) Oliver and Boyd, Edinburgh.

NILsson, E. 1951. Svensk Växtförädling 2. [see Lamprecht, H. 1968. Die neue Genekarte von Pisum und warum Mendel in seinen Erbsen-Kreuzungen keine Genekoppelung gefunden hat. Arb. Steiermärkischen Landesbibliothek Joanneum Graz, 10]

NOvirski, E., AND Blixt, s. 1976. Mendel and linkage. Science (in press).

sinNott, E. W., DunN, L. C., AND dobzhansky, T. 1958. Principles of Genetics, 5th edition. McGraw-Hill, Inc., N.Y.

WRIGHT, s. 1966. Mendel's ratios. In The Origin of Genetics, ed. C. Stern and Eva Sherwood, pp 173-175. W. H. Freeman and Co., San Francisco. 